ARTICLE

\title{
Central relay of bitter taste to the protocerebrum by peptidergic interneurons in the Drosophila brain
}

Sebastian Hückesfeld ${ }^{1}$, Marc Peters ${ }^{1} \&$ Michael J. Pankratz ${ }^{1}$

Bitter is a taste modality associated with toxic substances evoking aversive behaviour in most animals, and the valence of different taste modalities is conserved between mammals and Drosophila. Despite knowledge gathered in the past on the peripheral perception of taste, little is known about the identity of taste interneurons in the brain. Here we show that hugin neuropeptide-containing neurons in the Drosophila larval brain are necessary for avoidance behaviour to caffeine, and when activated, result in cessation of feeding and mediates a bitter taste signal within the brain. Hugin neuropeptide-containing neurons project to the neurosecretory region of the protocerebrum and functional imaging demonstrates that these neurons are activated by bitter stimuli and by activation of bitter sensory receptor neurons. We propose that hugin neurons projecting to the protocerebrum act as gustatory interneurons relaying bitter taste information to higher brain centres in Drosophila larvae.

\footnotetext{
${ }^{1}$ Department of Molecular Brain Physiology and Behavior, Life and Medical Sciences Institute (LIMES), University of Bonn, 53115 Bonn, Germany. Correspondence and requests for materials should be addressed to M.J.P. (email: pankratz@uni-bonn.de).
} 
$\mathrm{D}$ etailed knowledge exists on the anatomical distribution and function of gustatory receptors in mammals and Drosophila $^{1-5}$. In Drosophila 60 gustatory receptor genes encode 68 gustatory receptors ${ }^{6-8}$, with the majority of these receptors detecting bitter compounds ${ }^{9}$. Although gustatory receptors in Drosophila share no homology to mammalian taste receptors, the strategy used in both to detect a taste molecule, process its information and the valence of aversive bitter and appetitive sweet stimuli share similarities ${ }^{4}$. In contrast to the extensive knowledge on the peripheral coding of taste in flies and mammals, much less is known about the central pathways that relay and translate these signals into meaningful behaviour. Although broad regions in different parts of the brain have been shown to respond to various taste cues, there is little information on the molecular identity of specific neurons that convey different taste modalities to the higher brain centres ${ }^{10,11}$. Recently, secondary neurons that relay sweet taste from subesophageal zone (SEZ) to the antennal mechanosensory motor centre of adult Drosophila were characterized ${ }^{12}$. Analogous secondary neurons for other taste modalities have not yet been identified.

A candidate for conveying bitter taste from the SEZ to higher brain centres are neurons that express the hugin neuropeptide $^{13,14}$ (referred to as hugin neurons), whose arborizations in Drosophila larvae overlap with that of bitter gustatory receptor neurons (GRNs) expressing the caffeine receptor GR66 $a^{15-17}$. In adult Drosophila, GR66a was shown to represent a bitter receptor for detection of caffeine ${ }^{9,16,17}$ and inactivation of GR66a positive neurons leads to impairment of caffeine aversion ${ }^{17}$. In Drosophila a

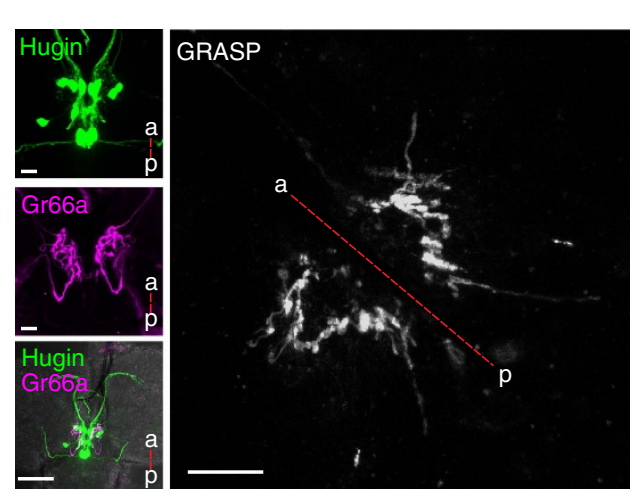

C

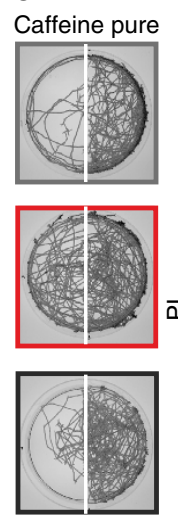

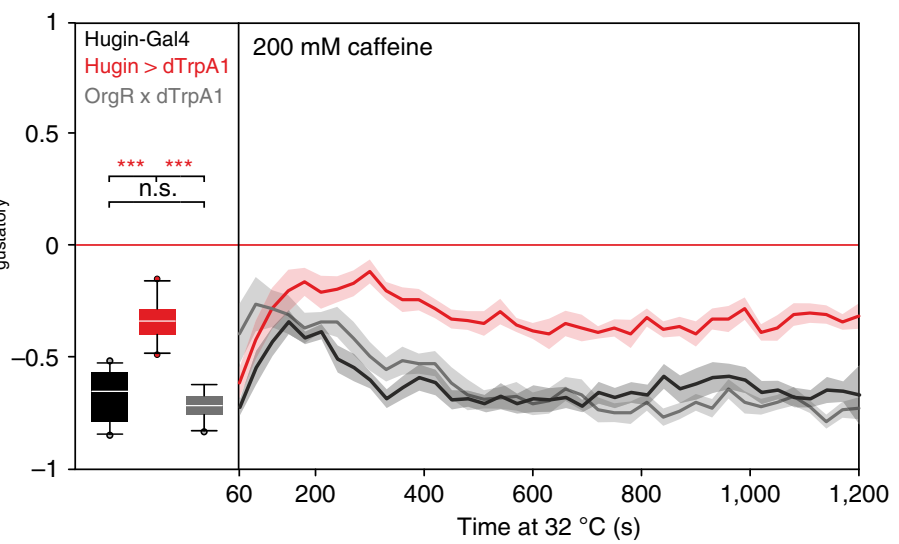

d

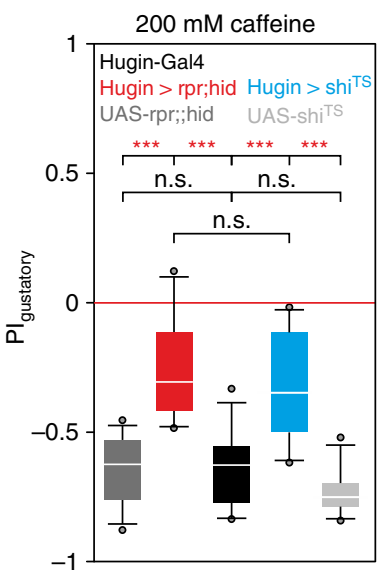

e

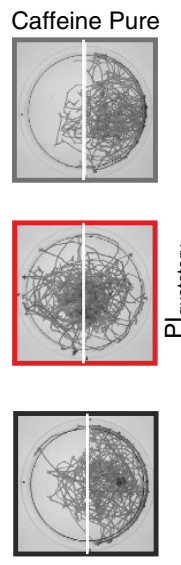

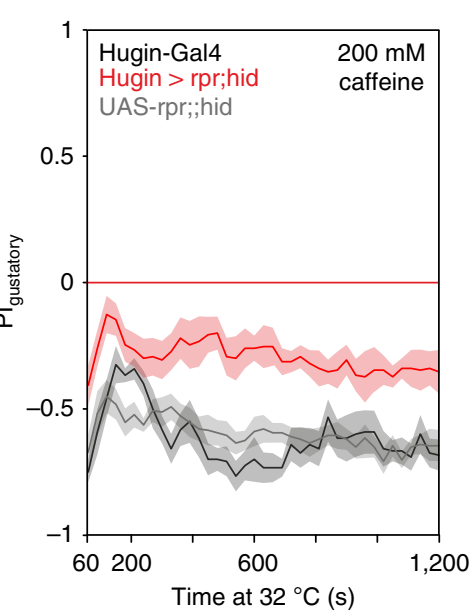

f

Caffeine Pure
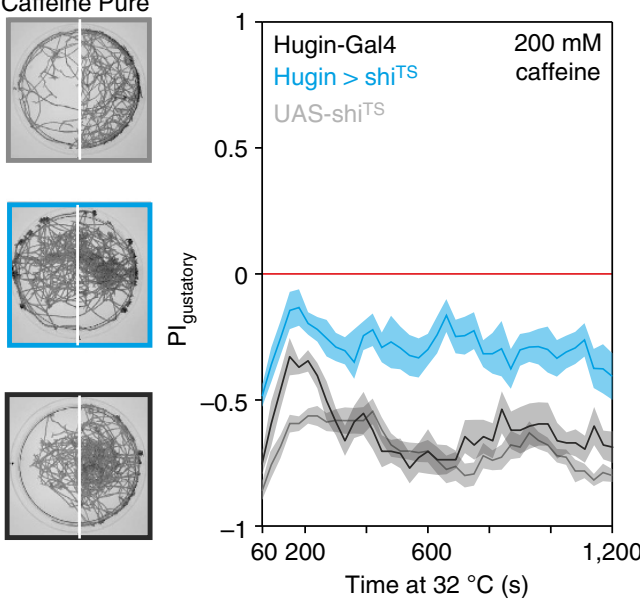

Figure 1 | Hugin neurons are part of bitter gustatory pathway. (a) Expression analysis of hugin neurons and GR66a positive dendrites in the SEZ using Hug-YFP;UAS-mRFP line crossed to GR66a-Gal4. Scale bars, $10 \mu \mathrm{m}$ for upper two panels and $50 \mu \mathrm{m}$ for lowest panel. (b) Close proximity of GR66a positive dendrites and hugin positive dendrites located in the SEZ using GRASP (Hug1.2lexA attp40 driving lexAop-CD4::spGFP11 crossed to GR66a-Gal4 driving UAS-CD4:::spGFP1-10). Scale bar, $10 \mu \mathrm{m}$. a-P = anterior-posterior. (c) Two-choice assay with $200 \mathrm{mM}$ caffeine. Left representative plates are time projections of the last $5 \mathrm{~min}$ of the 20 min experiment. Activating hugin neurons with UAS-dTrpA1 $(n=10)$ leads to impairment of choice behaviour $(P<0.001$, compared with the controls OrgR $\times \operatorname{dTrpA1}(n=10)$ and Hugin-Gal4 $(n=11)$, Mann-Whitney-U-Rank-Sum-Test (MWU-TEST)). Controls did not significantly differ from each other $(P=0.275, \mathrm{MWU}$-TEST). (d-f) Two-choice assay with $200 \mathrm{mM}$ caffeine. Ablation of hugin neurons by expression of UAS-rpr;;id ( $n=10)$ leads to impairment of bitter substrate avoidance compared with HugS3-Gal4 $(n=10)$ and UAS-rpr;; hid $(n=14)$ controls $(P<0.001$, MWU-Test). Controls did not differ from each other $\left(P=0.728\right.$, MWU-Test). Silencing hugin neurons by expression of UAS-shibire ${ }^{T S}$ ( $\left.n=10\right)$ shows same impairment on gustatory bitter choice compared with HugS3-Gal4 $(n=10)$ and UAS-shi ${ }^{\mathrm{TS}}(n=10)$ controls $(P<0.001, \mathrm{MWU}$-Test). Controls did not differ from each other $\left(P=0.168\right.$, UAS controls $P=0.082$, MWU-Test). All two-choice experiments were performed at $32^{\circ} \mathrm{C}$. Boxplots were generated from $P I$ values of the last $5 \mathrm{~min}$ of the 20 min experimental time. Significances are indicated as ${ }^{\star \star \star} P<0.001,{ }^{\star \star} P<0.01$ and ${ }^{\star} P<0.05$. Line plots showing the time course of the two choice experiments are displayed as mean (line) \pm s.e.m. (transparent areas). Details of descriptive statistics and statistics against chance levels for experimental lines are shown in Supplementary Table 1. 
a
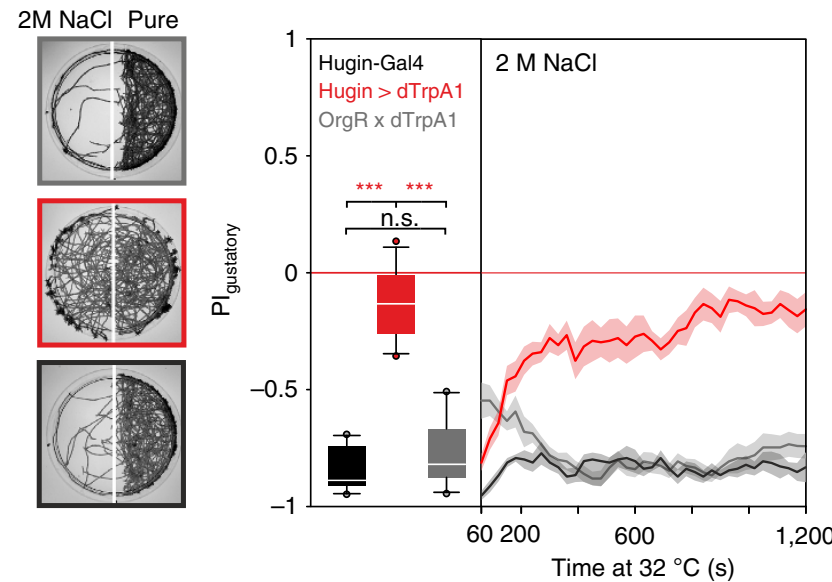

C

Fructose Pure

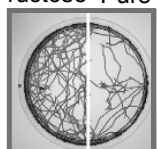

뭄

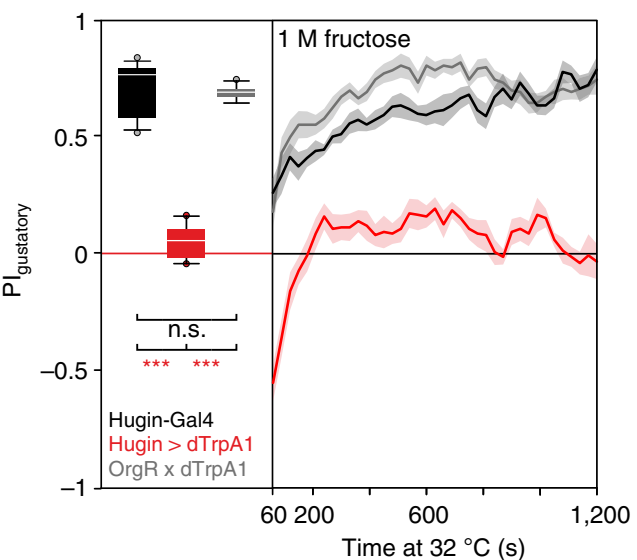

b

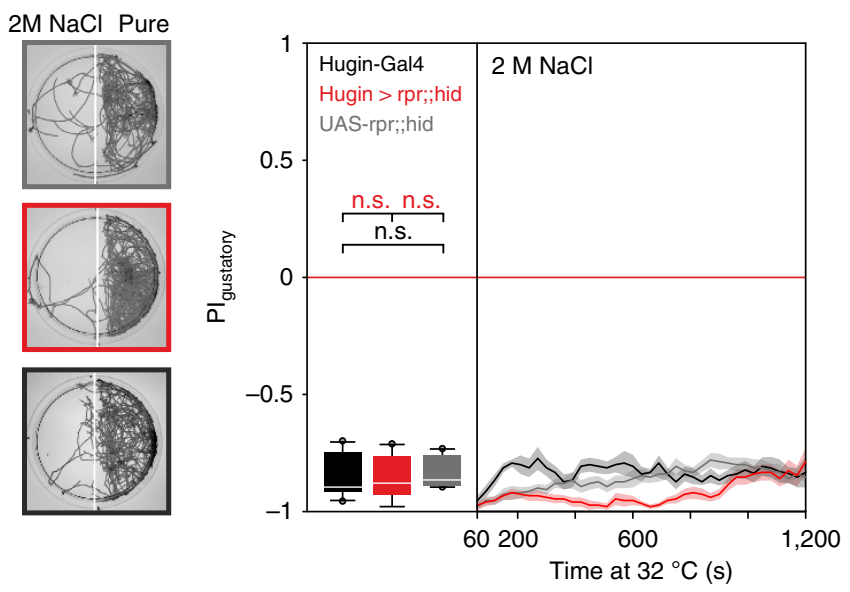

d

Fructose Pure
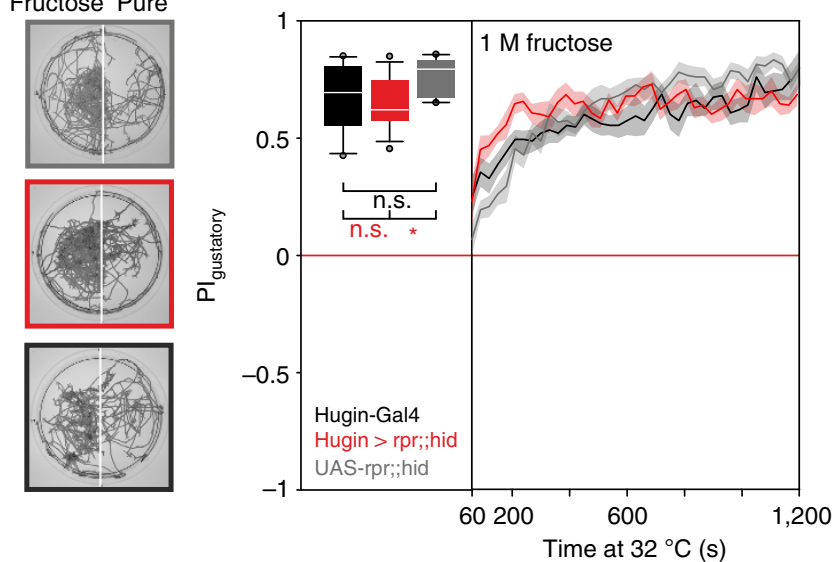

Figure 2 | Hugin neurons are not required for high salt or sweet taste processing. (a) Two choice assay with $2 \mathrm{M} \mathrm{NaCl}$. Activating hugin neurons with UAS-dTrpA1 $(n=11)$ leads to impairment of choice behaviour $(P<0.001$ compared with $\operatorname{OrgR} \times \mathrm{dTrpA} 1(n=10)$ and Hugin-Gal4 ( $n=10)$ controls, MannWhitney-U-Rank-Sum-Test (MWU-Test)). Controls did not differ from each other ( $P=0.241, \mathrm{MWU}$-Test). (b) No significant difference in avoidance behaviour was observed on $2 \mathrm{M} \mathrm{NaCl}$ between Hugin > rpr;;id $(n=10)$ and UAS-rpr;;-hid control $(n=10),(P=0.165$, MWU-Test $)$ or Hugin-Gal4 control $(n=10),(P=0.838$, MWU-TEST). Controls did not differ from each other $(P=0.241, \mathrm{MWU}$-Test). (c) Two choice assay with $1 \mathrm{M}$ fructose. Activating hugin neurons with UAS-dTrpA1 $(n=11)$ leads to impairment of choice behaviour $(P<0.001$ compared with OrgR $\times \mathrm{dTrpA} 1$ control $(n=11)$ and Hugin-Gal4 control $(n=13)$, MWU-Test). Controls did not differ from each other $(P=0.384, \mathrm{MWU}$-Test). (d) There was no significant difference on $1 \mathrm{M}$ fructose choice behaviour between Hugin > rpr; ;hid $(n=12)$ and Hugin-Gal4 control $(n=10)(P=0.668$, MWU-Test). UAS-rpr;;hid control larvae $(n=10)$ showed significant difference to Hugin $>$ rpr;;ihid $(P=0.013$, MWU-Test). Controls did not differ from each other $(P=0.104$, MWU-Test). Sample two choice plates are shown on the left side of each experiment for each genotype for the last $5 \mathrm{~min}$ of the experiment. Boxplots were generated from $P I$ values of the last $5 \mathrm{~min}$ of the $20 \mathrm{~min}$ experiment. Significances are indicated as ${ }^{\star \star \star} P<0.001,{ }^{\star} * P<0.01$ and ${ }^{\star} P<0.05$. Line plots showing the time course of the two choice experiments are displayed as mean (line) \pm s.e.m. (transparent areas). Details of descriptive statistics and statistics against chance levels for experimental lines are shown in Supplementary Table 2.

larvae, artificial activation of GR66a positive neurons leads to aversive behaviour ${ }^{18}$. Thus, hugin neurons were good candidates for acting as a central relay for bitter information from sensory neurons.

Using classical two-choice behavioural experiments, electrophysiological measurements as well as calcium imaging analysis, we now show that hugin neurons relay caffeine as well as other bitter taste signals from sensory neurons to the protocerebrum, acting as bitter taste interneurons in the Drosophila larval brain.

\section{Results}

Hugin neurons are required for caffeine avoidance response. We first asked whether the hugin neurons make contacts with caffeine responsive GR66a neurons. Using the GFP reconstitution across synaptic partners (GRASP) approach ${ }^{19}$, we could indeed observe a GRASP signal in the SEZ, indicating that caffeine receptor neurons and hugin neurons are in close proximity to each other (Fig. 1a,b).

Activating the hugin neurons causes the larvae to stop feeding and move out of a strongly appetitive food source (yeast) ${ }^{20}$, which could be due to activation of an aversive bitter taste pathway. We therefore tested the behavioural response to caffeine in a two choice assay. When hugin neurons are activated with the temperature sensitive cation channel dTrpA1, the animals showed impaired aversion to caffeine stimuli (Fig. 1c). We next asked how the animals would behave if hugin neuronal activity was suppressed, by ablating the hugin neurons. These animals showed significantly less avoidance to caffeine (Fig. 1d,e). To exclude potential developmental effects, we also expressed the temperature sensitive mutant form of dynamin (shibire ${ }^{\mathrm{TS}}$ ) in the hugin neurons, which leads to a block of synaptic release in a 
temperature dependent manner. The loss of proper bitter aversion still persisted (Fig. 1d,f).

We next asked if hugin neurons were involved in other taste modalities. We first tested high salt $(2 \mathrm{M} \mathrm{NaCl})$, which is very aversive for larvae ${ }^{21}$, in two-choice assays. As with caffeine, activation of the hugin neurons led to animals showing impaired aversion to high salt (Fig. 2a). However, no difference to control was observed when hugin neurons were ablated, indicating that hugin neurons are not required for the behavioural response to high salt (Fig. 2b). High caffeine and salt levels are both aversive gustatory stimuli. To determine how manipulation of hugin neuronal activity affected the response to an appetitive cue, we performed two-choice assays with $1 \mathrm{M}$ fructose. In this case, activation led to impaired attraction to fructose, whereas ablation had no effect relative to control (Fig. 2c,d; Supplementary Fig. 1). Thus, when hugin neurons are activated, the larvae are no longer able to respond properly to different taste modalities, such as yeast protein ${ }^{20}$, fructose, high salt and caffeine. This chemosensory response was specific for taste, as olfactory behaviour was not affected (Fig. 3), showing that activation of hugin neurons selectively disrupts gustatory chemosensory processing. However, inhibiting the hugin neurons results in the inability to respond appropriately to caffeine, indicating that hugin neurons process bitter taste information. In sum, when hugin neurons are activated, larvae perceive all tested substrates (water, caffeine, salt and fructose) as bitter tasting. In contrast, when hugin neurons are inactivated, only the bitter substrate choice is affected since proper detection of bitter compounds no longer occurs.

Distinct hugin neurons modulate taste and feeding behaviour. As the hugin neuronal cluster is composed of different classes with different projection targets ${ }^{13,14}$, we next wanted to determine which class was responsible for taste processing and feeding regulation. Through promoter deletion analysis, we generated a hugin promoter-Gal4 line which showed target gene expression exclusively in the eight hugin cells (four per hemisphere) that project to the protocerebrum (HugPC-Gal4, Fig. 4a). To see whether this subset of hugin neurons (huginPC neurons) is necessary for proper bitter taste processing, we ablated these neurons (Fig. 4b) and tested larvae in the caffeine two-choice experiment. Ablation of the huginPC neurons resulted in the inability to appropriately avoid caffeine as compared with control animals (Fig. 4c). Consistent with the ablation of all hugin neurons (Fig. 2), ablating just the huginPC neurons had no effect compared with controls on high salt or fructose (Fig. 4d,e, controls are re-plotted from Fig. 2b,d). We note that, in the case of $2 \mathrm{M} \mathrm{NaCl}$, there was a significant difference to one of the controls (UAS-rpr;; hid control larvae). Therefore, we additionally tested the animals on a lower, but still aversive salt concentration $(500 \mathrm{mM})$, which showed that huginPC neurons are not necessary for proper high salt aversion (Supplementary Fig. 2, for descriptive statistics see Supplementary Table 7). Activating just the huginPC neurons was sufficient to suppress food intake, induce wandering-like behaviour (Fig. 5a; Supplementary Fig. 3), as well as decrease the motor pattern of pharyngeal muscle contractions (Fig. 5b). These results indicated that the hugin neurons responsible for feeding and caffeine taste mediated behaviours are those that project to the protocerebrum.

Activity of huginPC neurons upon bitter stimulation. To further investigate the connection between bitter taste and hugin neurons, we asked if the huginPC neurons could be activated by caffeine using CaMPARI (Calcium Modulated Photoactivatable Ratiometric Integrator), that allows monitoring a

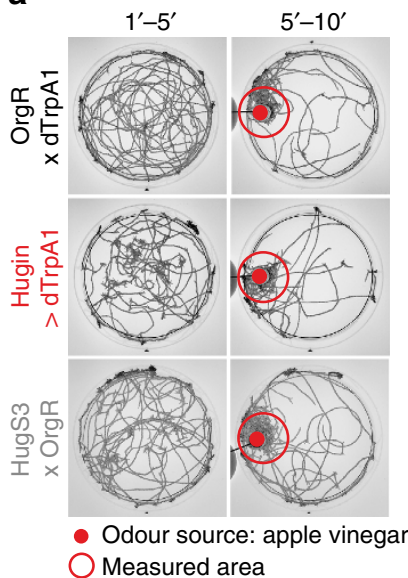

b

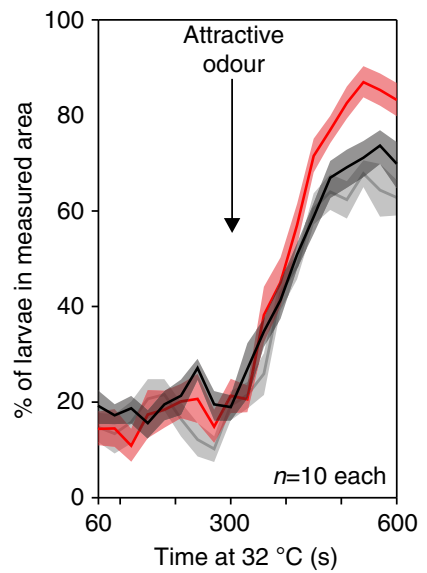

Figure 3 | Activation of hugin neurons does not impair attractive

olfactory guidance. (a) Olfactory assay for choice behaviour in response to an attractive odour (apple vinegar). Shown are time projections of the pure agar plates for the first $5 \mathrm{~min}$ of the experiment and the last $5 \mathrm{~min}$ of the experiment with the appetitive odour source apple vinegar. (b) Larvae of all three geneotypes (OrgR $\times$ dTrpA1, Hugin $>d \operatorname{TrpA} 1$ and HugS3 $\times$ OrgR) detected the appetitive odour at the same timepoint and were equally fast at reaching the nearby area beneath the apple vinegar spot above the plate.

of calcium activity in intact animals. Calcium activity and simultaneous presence of ultraviolet-light $(405 \mathrm{~nm})$ lead to an irreversible conversion from green to red fluorescence of the neurons of interest $\mathrm{t}^{22}$. When we placed intact larvae in solutions containing water and water mixed with caffeine, fructose, high $\mathrm{NaCl}$ or yeast (Fig. 6a), huginPC neurons were strongly activated by caffeine (Fig. 6b,c). They also showed a concentration dependent increase in calcium activity with increasing caffeine concentrations (Fig. 6d). Other bitter tastants, such as quinine and denatonium, also activated huginPC neurons (Fig. 6e). We tested the behavioural relevance for this rise in calcium activity for denatonium in two-choice experiments, since denatonium led to robust avoidance behaviour in control larvae at $32{ }^{\circ} \mathrm{C}$ (experimental temperature). The calcium activity in huginPC neurons was behaviourally relevant since ablation of these neurons resulted in larvae with impaired aversion to the taste of denatonium (Fig. 6f) (no difference when tested against chance levels, see Supplementary Table 5). Unexpectedly, we observed decrease in huginPC calcium activity in larvae placed in high salt, fructose and yeast relative to water alone (Fig. 6c). Although the functional significance of this repression is not clear, it has been shown that bitter taste pathways can inhibit sweet pathways in adult $^{23}$ and larval ${ }^{24}$ Drosophila, reflecting an interaction between pathways involving different taste modalities. This crossregulation might be a general strategy to efficiently detect distinct taste modalities, since in case of the huginPC neurons, a sweet taste stimulus would inhibit the activity of bitter interneurons.

Finally we asked if there is a functional connection, in addition to the anatomical connection (Fig. 1a,b), between the caffeine sensing GR66a neurons and hugin neurons. To this end, we activated the GR66a neurons and then monitored the activity of hugin neurons using the calcium indicator GCaMP6s (Fig. 7a,b). Activation of GR66a by dTrpA1 and simultaneous calcium imaging of huginPC projections resulted in the induction of rhythmically occurring calcium peaks, demonstrating a functional connection between GR66a and huginPC neurons (Fig. 7b). 
a

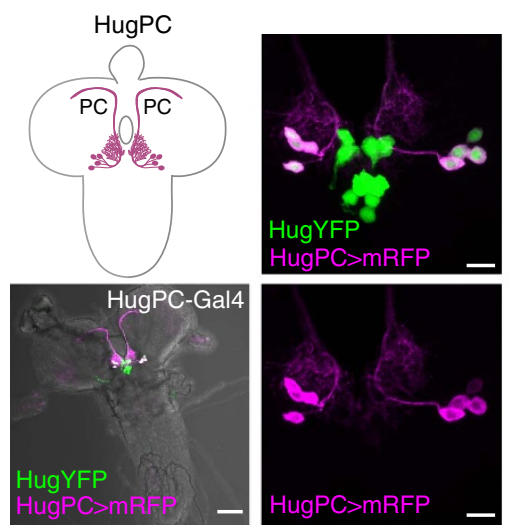

b

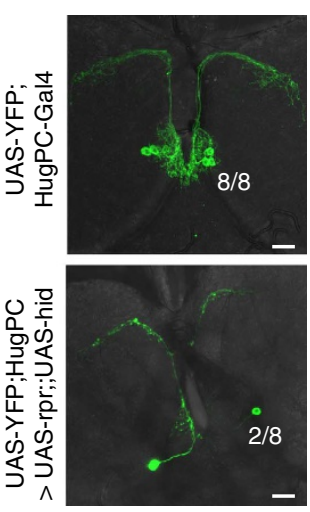

d

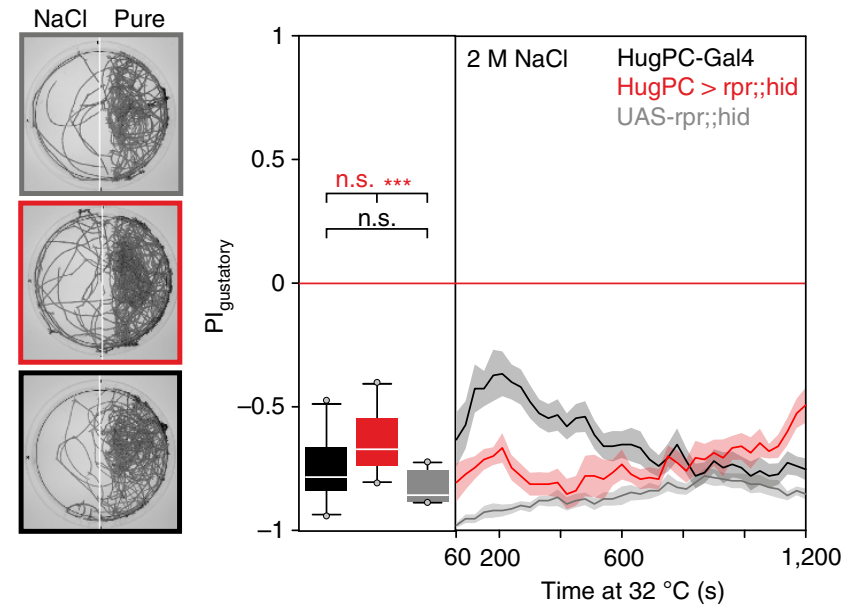

c

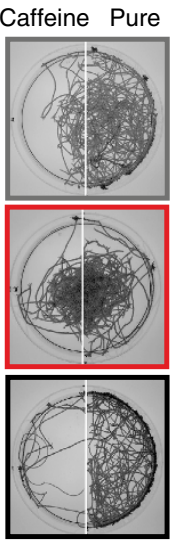

e Fructose Pure

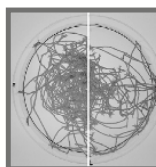$$
\frac{1}{2}
$$

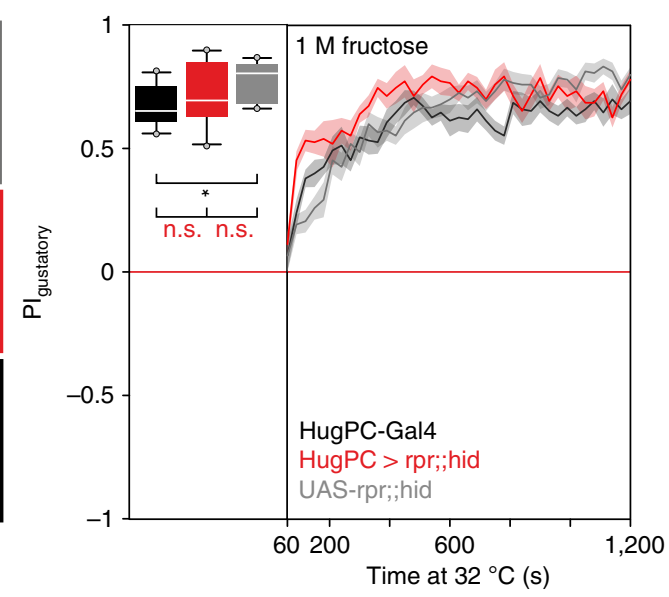

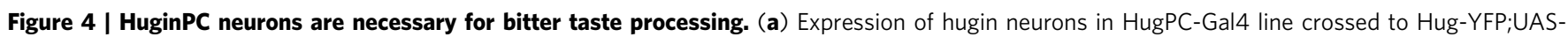
mRFP line. Eight hugin neurons that project to the protocerebrum are labelled by UAS-mRFP. Scale bars, $50 \mu \mathrm{m}$ for left and $10 \mu \mathrm{m}$ for right panels. (b) In HugPC > rpr;; hid larvae 6 of 8 huginPC neurons were ablated successfully $(n=17) ; 2$ ( \pm 0.3 SE). Scale bars: $20 \mu \mathrm{m}$. (c) Two choice assay with caffeine after ablation of huginPC neurons with UAS-rpr;,;hid ( $n=13),(P<0.001$ compared with HugPC-Gal4 control ( $n=10)$ and UAS-rpr;;, hid control $(n=14)$, Mann-Whitney-U-Rank-Sum-Test (MWU-Test)). Controls did differ from each other $(P=0.006, M W U-T e s t)$. (d) Two-choice assay with 2 M $\mathrm{NaCl}$. Ablating huginPC neurons with UAS-rpr;"hid $(n=10)$ causes no impairment of high salt avoidance compared with HugPC-Gal4 control ( $n=10)$ $(P=0.076$, MWU-Test). A significant difference in salt avoidance occurred comparing HugPC $>$ rpr;;hid with UAS-rpr;;hid larvae ( $n=10, P<0.001$, MWU-Test). Controls did not differ from each other ( $P=0.167, \mathrm{MWU}$-Test). (e) Two-choice assay with $1 \mathrm{M}$ fructose. Ablating huginPC neurons with UAS-rpr;; hid $(n=10)$ causes no impairment in fructose attraction compared with HugPC-Gal4 control ( $n=10, P=0.241$, MWU-Test) or UAS-rpr;; hid control ( $n=10, P=0.450$, MWU-Test). Controls show significant difference to each other $(P=0.013, M W U$-Test). Boxplots were generated from PI values of the last $5 \mathrm{~min}$ of the 20 min experiment time in two-choice assays. Significances are indicated as ${ }^{\star \star \star} P<0.001$, ${ }^{\star \star} P<0.01$ and ${ }^{\star} P<0.05$. Line plot shows the time course of the two choice experiment displayed as mean (line) \pm s.e.m. (transparent areas). Details of descriptive statistics and statistics against chance levels for experimental lines are shown in Supplementary Table 3.

\section{Discussion}

The bitter taste rejection response is important for all animals that encounter toxic or harmful food in their environment. Here we showed that the hugin neurons in the Drosophila larval brain function as a relay between bitter sensory neurons and higher brain centres (Fig. 8). Strikingly, activation of the hugin neurons made the animals significantly more insensitive to substrates with negative valence like bitter (caffeine) and salt (high $\mathrm{NaCl}$ ), as well as positive valence like sweet (fructose). In other words, when the hugin neurons are active these animals 'think' they are tasting bitter and therefore become insensitive to other gustatory cues. This is in line with observations made in mice, in which optogenetically activating bitter cortex neurons caused animals to avoid an empty chamber illuminated with blue light. In this situation, although mice do not actually taste something bitter, they avoid the empty chamber since the bitter perception has been optogenetically induced in the central nervous system (CNS) and the mice 'think' they are tasting a bitter substance ${ }^{25}$.

In our previous work, activation of all hugin neurons led to behavioural and physiological phenotypes such as decreased feeding, decrease in neural activity of the antennal nerve (AN), and induction of a wandering-like behaviour ${ }^{20}$. We have now pinpointed the neurons responsible specifically to those that project to the protocerebrum. These neurons not only respond to bitter stimuli, but also show a concentration dependent increase in calcium activity in response to caffeine. Dose dependent coding of bitter taste stimuli was previously shown to occur in peripheral bitter sensory neurons, where bitter sensilla exhibit dose dependent responses to various bitter compounds ${ }^{9}$. Larvae in which the huginPC neurons have been ablated still showed some 
a

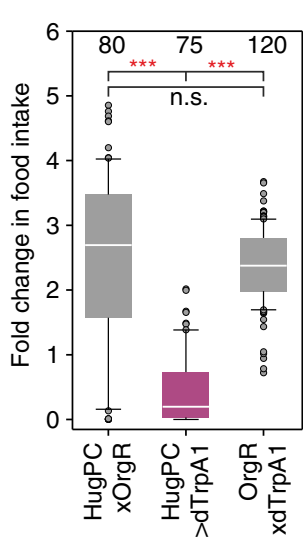

b

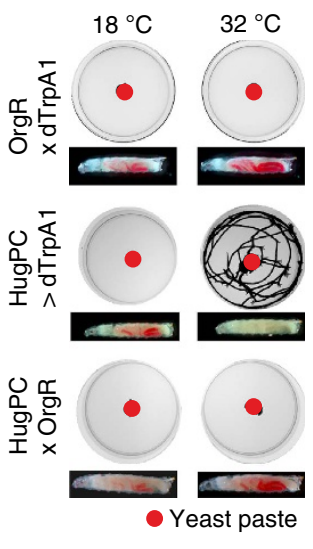

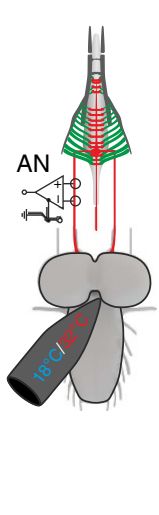
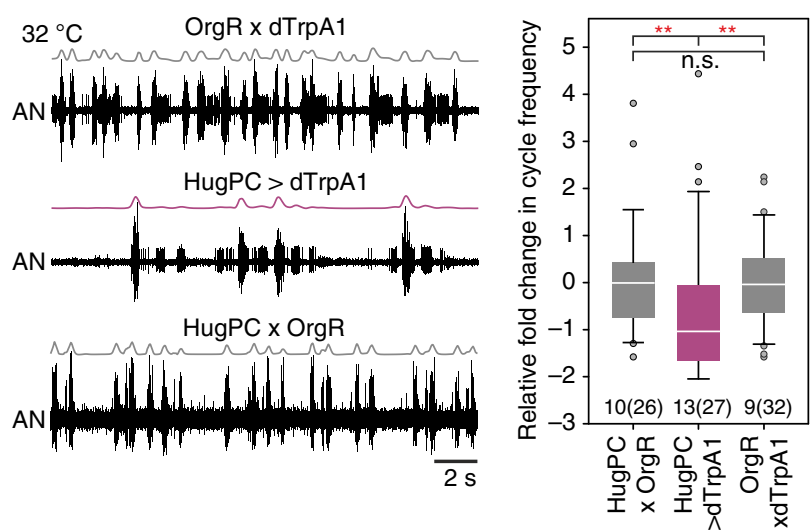

Figure 5 | HuginPC neurons modulate feeding and wandering like behaviour. (a) HugPC-Gal4 line driving UAS-dTrpA1 ( $n=75$ larvae). Larvae show reduction in food intake compared with $\operatorname{OrgR} \times \operatorname{dTrpA1}(n=120$ larvae $)$ and HugPC $\times \operatorname{OrgR}(n=80$ larvae $)(P<0.001$, MWU-Test). Controls did not differ from each other $(P=0.107, \mathrm{MWU}$-Test). Activation of huginPC neurons with dTrpA1 induces wandering like behaviour, where larvae leave the appetitive food source yeast. Shown are time projections over $20 \mathrm{~min}$ of plates with apple juice agar and a red spot of yeast in the middle. Decrease of food intake was measured as \% of red stained gut content compared with the area of the whole larva. (b) Extracellular recordings of the antennal nerve (AN). Activation of huginPC neurons with UAS-dTrpA1 ( $n=13$ larvae, 27 temperature steps) leads to significant decrease in cycle frequency of the AN motor pattern compared with $\operatorname{OrgR} \times \mathrm{dTrpA} 1$ control ( $n=9$ larvae, 32 temperature steps) and HugPC $\times$ OrgR control ( $n=10$ larvae, 26 temperature steps) ( $P=0.003$, MWU-Test). Controls did not differ from each other. Significances are indicated as ${ }^{\star \star \star} P<0.001,{ }^{\star \star} P<0.01$ and ${ }^{\star} P<0.05$. Details of descriptive statistics are shown in Supplementary Table 4.

avoidance to caffeine. Whether this implies the existence of other interneurons being involved in caffeine taste processing remains to be determined. Interestingly, the huginPC neurons are inhibited when larvae taste other modalities like salt $(\mathrm{NaCl})$, sugar (fructose) or protein (yeast). This may indicate that taste pathways in the brain are segregated, but influence each other, as previously suggested ${ }^{10}$.

Bitter compounds may be able to inhibit the sweet-sensing response to ensure that bitter taste cannot be masked by sweet tasting food. This provides an efficient strategy for the detection of potentially harmful or toxic substances in food $^{26,27}$. For appetitive tastes like fructose and yeast, bitter interneurons neurons like the huginPC neurons in the CNS may become inhibited to ensure appropriate behaviour to pleasant food. Salt is a bivalent taste modality, that is, low doses of salt drive appetitive behaviour, whereas high doses of salt are aversive to larval ${ }^{21,28}$ and adult ${ }^{29,30}$ Drosophila. Inhibition of huginPC neurons when larvae are tasting salt might be due to a different processing circuit for different concentrations of salt and the decision to either take up low doses or reject high doses.

Taken together, we propose that hugin neuropeptide neurons projecting to the protocerebrum represent a hub between bitter gustatory receptor neurons and higher brain centres that integrate bitter sensory information in the brain, and through its activity, influences the decision of the animal to avoid a bitter food source. The identification of second order gustatory neurons for bitter taste will not only provide valuable insights into bitter taste pathways in Drosophila, but may also help in assigning a potentially novel role of its mammalian homologue, Neuromedin $\mathrm{U}$, in taste processing.

\section{Methods}

Fly lines. Wild type (OrgR) crossed to UAS-dTrpA1 (Bloomington \#26263) served as control in Figs 1, 2, 3 and 5. Hugin-Gal4 (HugS3-Gal4 (ref. 13), Bloomington\# 58769), GR66a-Gal4 (second Chr., gift from K.Scott ${ }^{31}$ (formerly described as GR66C1)), GR66a-Gal4 (third Chr., Bloomington\# 57670 used in Fig. 7b), HuginlexA (Hug1.2-lexA attp40 (ref. 32)), HugPC-Gal4 (see generation of this Gal4-line below), UAS-eNpHR-YFP (Bloomington\# 41753, referred here as UAS-YFP in Supplementary Fig. 3, since this homozygous line together with HugPC-Gal4 was used as fluorescent marker only), UAS-CaMPARI (Bloomington \#58761), UAS-rpr;;UAS-hid (UAS-rpr (Bloomington\# 5823) crossed homozygous to

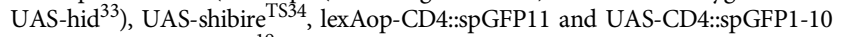
were gifts from K. Scott ${ }^{19}$, 13x LexAop2-IVS-GCamP6s-p10 (Bloomington \#44274).

Fly care. Adult flies and larvae were kept on $25^{\circ} \mathrm{C}$ under $12 \mathrm{~h} \mathrm{light/dark} \mathrm{condi-}$ tions. For electrophysiological and food intake experiments $4 \mathrm{~h}$ egg collections were made on apple juice agar plates containing a spot of yeast-water paste. After $48 \mathrm{~h}$, larvae were transferred into food vials containing lab standard fly food. For other experiments (two-choice, CaMPARI, GCamP) larvae were raised in vials containing standard fly food with a spot of yeast for 4 days. All larvae used for the experiments were $98 \pm 2 \mathrm{~h}$ old. Only feeding third instar larvae were used for the experiments.

Generation of transgenic flies. For HugPC-Gal4 line, a 544 bp Hugin fragment $155 \mathrm{bp}$ upstream of the ATG was amplified with primerl (AAG GGT TTG GTT TAA TTT ATT TAT GTC ATA) and primer2 (GAG CCT GAT TAG GTC CCT GAT GTT TAA ACT T) and cloned into pCaSpeR-AUG-Gal4-X vector (Addgene plasmid 8,378 . The construct was injected into $\mathrm{w}^{[1118]}$ ).

Two-choice gustatory assay. To measure the preference index of larvae towards given appetitive or aversive substrates, $9 \mathrm{~mm}$ diameter petri dishes were filled with $20 \mathrm{ml}$ warm water agar (2.125\% Agar-Agar, Kobe). After $20 \mathrm{~min}$ of air drying half of the agar was cut away and discarded. Compounds $(2 \mathrm{M} \mathrm{NaCl}, 1 \mathrm{M}$ Fructose, $200 \mathrm{mM}$ Caffeine, $10 \mathrm{mM}$ Denatonium) were diluted in warm agar in the given concentration until the agar fluid was clear, and filled in the other half of the petri dish $(10 \mathrm{ml})$. After air drying again for $20 \mathrm{~min}$, petri dishes were prewarmed in the $32^{\circ} \mathrm{C}$ incubator $1.5 \mathrm{~h}$ prior to the experiment. All two-choice experiments were performed at $32{ }^{\circ} \mathrm{C}$ for comparability between all genotypes. For each experiment 30 larvae were taken out of standard fly food and washed with tap water. Larvae were then placed on the water side of the two-choice dishes and videotaped for 20 min. Videos were processed with FIJI (ImageJ) and analysed using a custom written script for FIJI. Analysis of PI values started $60 \mathrm{~s}$ after the start of the experiment to ensure proper tracking of larvae due larval accumulation at the beginning by placing them on the pure agar side. $\mathrm{PI}_{\text {gustatory }}$ was calculated as (\#larvae $_{\text {substrate }}-$ \#larvae $_{\text {water }}$ )/\#larvae total. $_{\text {. }}$.

One-choice olfactory assay. For testing response to an attractive odour (apple vinegar), a one-choice assay was performed. Agar plates were used (as described earlier for two-choice petri dishes) and placed into the incubator at $32^{\circ} \mathrm{C}$ for $1.5 \mathrm{~h}$. Larvae were videotaped at $32^{\circ} \mathrm{C}$ for $5 \mathrm{~min}$, and then an Eppendorf cup $(1.5 \mathrm{ml})$ with filter paper soaked with apple vinegar was placed on one side of the water agar plate above the larvae such that they were not able to reach the odorant source. Movement of larvae was analysed using the custom made FIJI macro. 
a

Hugin-Gal4 >
UAS-CaMPARI

2 min in substance

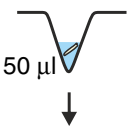

30 s UV light

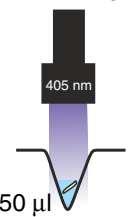

b
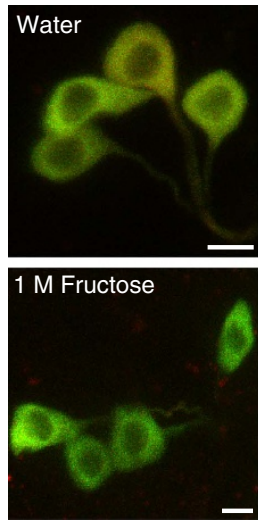

HugPC neurons
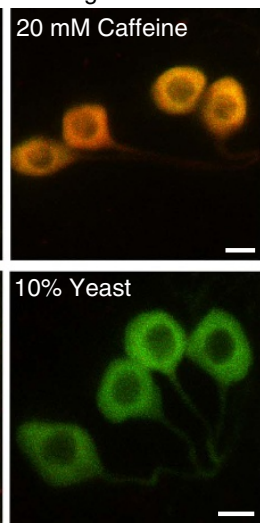

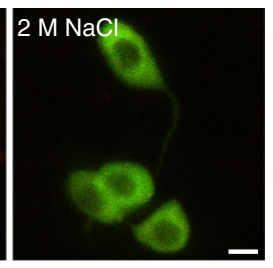

No UV light

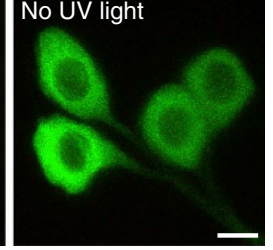

C

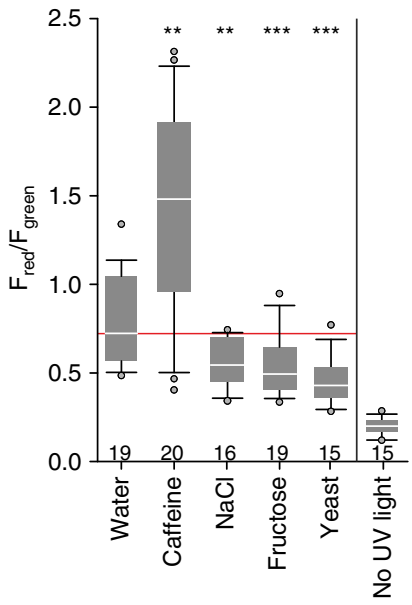

d

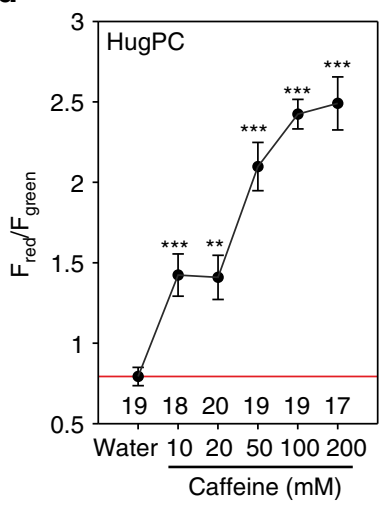

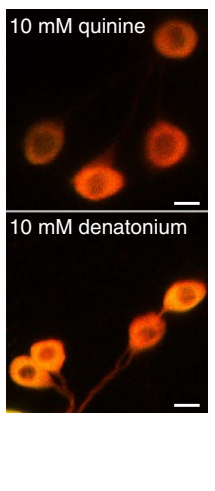

f

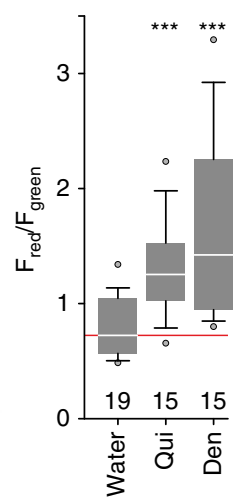

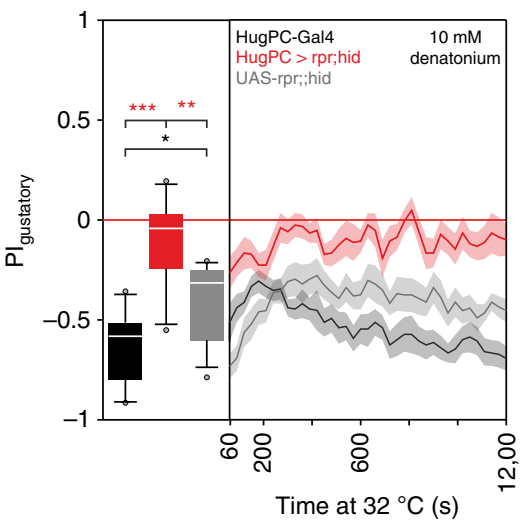

Figure 6 | HuginPC neurons relay bitter taste information. (a) Experimental setup (left panel). (b) Larvae expressing UAS-CaMPARI in huginPC neurons showed green to red photoconversion only when larvae were placed in caffeine solution. Scale bars, $5 \mu \mathrm{m}$. (c) Quantification of $F_{\text {red }}$ divided by $F_{\text {green }}$ for $z$-projections of huginPC neurons $(P=0.003$ for caffeine, $P=0.004$ for $\mathrm{NaCl}, P<0.001$ for fructose and $P<0.001$ for yeast mixed in water compared with pure water, Mann-Whitney-U-Rank-Sum-Test (MWU-Test), $n$ of larvae displayed beneath boxplots). Right most boxplot shows fluorescence ratio of huginPC neurons without ultraviolet light exposure $(P<0.001, \mathrm{MWU}$-Test). (d) Measurement of huginPC neurons expressing UAS-CaMPARI in larvae confronted with different concentrations of caffeine. HuginPC neurons display increasing red/green ratio with increasing caffeine concentration (10 mM: $P<0.001,20 \mathrm{mM}: P=0.003,50 \mathrm{mM}: P<0.001,100 \mathrm{mM}: P<0.001,200 \mathrm{mM}: P<0.001$ compared with water alone, MWU-Test). Dots represent mean, whiskers represent s.e.m. (e) HuginPC neurons expressing UAS-CaMPARI display high calcium activity in $10 \mathrm{mM}$ quinine $(P<0.001, \mathrm{MWU}$-Test) or $10 \mathrm{mM}$ Denatonium $(P<0.001, \mathrm{MWU}$-Test). numbers beneath boxplots indicate number of larvae used for each experiment. Scale bars: $5 \mu \mathrm{m}$. (f) Two-choice assay with $10 \mathrm{mM}$ denatonium. Ablating huginPC neurons with UAS-rpr;;i, $(n=10)$ leads to impairment of gustatory choice on denatonium compared with HugPC-Gal4 control ( $n=10, P<0.001$, MWU-Test) or UAS-rpr;;i,id control ( $n=12, P=0.002$, MWU-Test). Controls show significant difference to each other $(P=0.027, \mathrm{MWU}$-Test). Boxplots were generated from $P I$ values of the last $5 \mathrm{~min}$ of the 20 min experiment time for two-choice experiment. Significances are indicated as ${ }^{\star \star \star} P<0.001,{ }^{\star \star} P<0.01$ and ${ }^{\star} P<0.05$. Line plots showing the time course of the two choice experiment and dot plots are displayed as mean (line) \pm s.e.m. (transparent areas). Details of descriptive statistics and statistics against chance levels for experimental lines are shown in Supplementary Table 5.

Food intake assay. Apple juice agar plates were prepared with a spot of red yeast paste in the middle of the plate. Plates were then placed in incubators precooled to $18{ }^{\circ} \mathrm{C}$ or prewarmed to $32^{\circ} \mathrm{C}$ for $2 \mathrm{~h}$. After $30 \mathrm{~min}$ starvation, five larvae were transferred on top of the yeast paste of a plate and videotaped for $20 \mathrm{~min}$. After 20 min of videotaping, larvae were transferred into a small cell strainer and washed with $60^{\circ} \mathrm{C}$ hot water. Larvae were then transferred to glass slides for photo documentation and analysed with the open source software FIJI (ImageJ) and a custom written analysis macro, which calculated the percentage of the red stained surface of the body compared with the whole body of the larva. To calculate the fold change of food intake from 18 to $32^{\circ} \mathrm{C}$, the value of all $32^{\circ} \mathrm{C}$ values was divided by the mean value of all $18^{\circ} \mathrm{C}$ values.

Electrophysiology. Third instar larvae were dissected in $35 \mathrm{~mm}$ petri dishes coated with $5 \mathrm{ml}$ two-component silicone (Elastosil RT). Larvae were pinned down dorsal side up at the anterior and posterior end using $77 \mu \mathrm{m}$ thick sharp etched tungsten needles. The larva was cut open longitudinally along the dorsal midline and the cuticle was pinned aside with $40 \mu \mathrm{m}$ tungsten needles. Interior organs like fat body, intestine or salivary glands were removed except for the cephalopharyngeal skeleton and CNS with attached nerves of interest. Eye and leg imaginal discs were also removed. A transversal cut of the cuticle was performed beneath the CNS to reveal the AN. Nerves not needed for the respective recording were cut. A piece of thinned Parafilm was placed beneath the nerve of interest. This nerve was isolated from the surrounding solution with two adjacent jelly pools. Motor output of the AN was measured using custom made silver wire electrodes connected to a preamplifier (Model MA103, Ansgar Büschges group electronics lab). The preamplifier was connected to a four-channel amplifier/signal conditioner (Model MA 102, Ansgar Büschges group electronics lab). All recorded signals were amplified (amplification factor: 5,000) and filtered (bandpass: $0.1-3 \mathrm{kHz}$ ). Recordings were sampled at $20 \mathrm{kHz}$. Data were acquired with Micro3 1,401 A/D board (Cambridge Electronic Design) and Spike2 software (Cambridge Electronic Design).

Calcium imaging with CaMPARI. High-power LED of $405 \mathrm{~nm}$ (Thorlabs, M405L2-UV (405 nm) Mounted LED, 1,000 mA, $410 \mathrm{~mW}$ ) was driven with a LED 
a

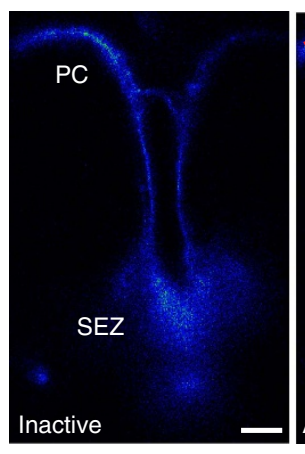

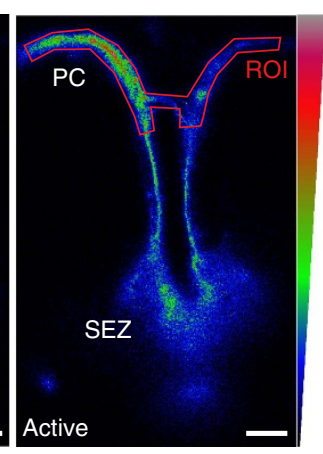

b
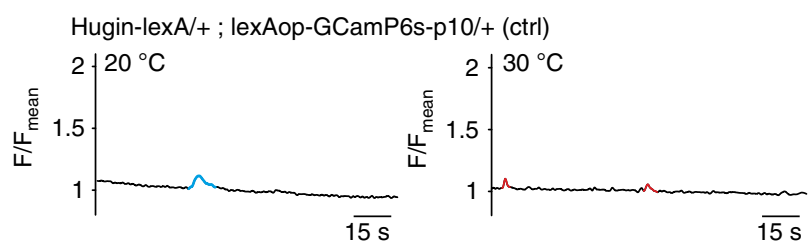

UAS-dTrpA1/Hugin-lexA ; GR66a-Gal4/lexAop-GCamP6s-p10 (exp)

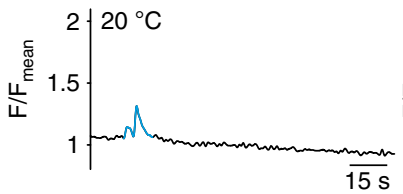

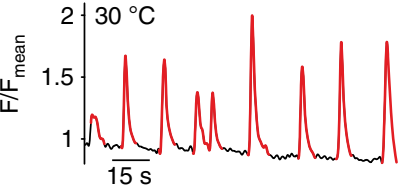

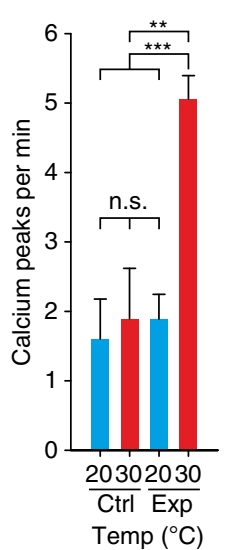

Figure 7 | HuginPC neurons are functionally connected to GR66a neurons. (a) Example of huginPC neuronal arborizations in the protocerebrum expressing GCamP6s in inactive and active state. Scale bars, $20 \mu \mathrm{m}$. (b) Activation of GR66a neurons by dTrpA1 in larvae expressing Hugin-lexA-lexAopGCamP6s. Sample traces of calcium currents show rhythmic activity of huginPC projections when GR66a neurons are activated at $30^{\circ} \mathrm{C}$. Quantification of calcium spikes per min showed no significant difference between control (ctrl, $n=13)$ and experimental genotypes $(\exp , n=14)$ at $20^{\circ} \mathrm{C}(P=0.241$, Mann-Whitney-U-Rank-Sum-Test (MWU-Test); control at 20 and $30^{\circ} \mathrm{C}(n=13),\left(P=0.790, \mathrm{MWU}\right.$-Test); and control at $30^{\circ} \mathrm{C}$ and experimental genotype at $20^{\circ} \mathrm{C}\left(P=0.197, \mathrm{MWU}\right.$-Test). Significant difference could be shown for the experiment at 20 and $30^{\circ} \mathrm{C}(n=14)(P=0.004$, MWU-Test), as well as control at $20^{\circ} \mathrm{C}$ compared with experiment at $30^{\circ} \mathrm{C}\left(P<0.001, \mathrm{MWU}\right.$-Test). Scale bars, $20 \mu \mathrm{m}$. Significances are indicated as ${ }^{\star \star \star} P<0.001,{ }^{\star \star} P<0.01$ and ${ }^{\star} P<0.05$. Details of descriptive statistics are shown in Supplementary Table 6.

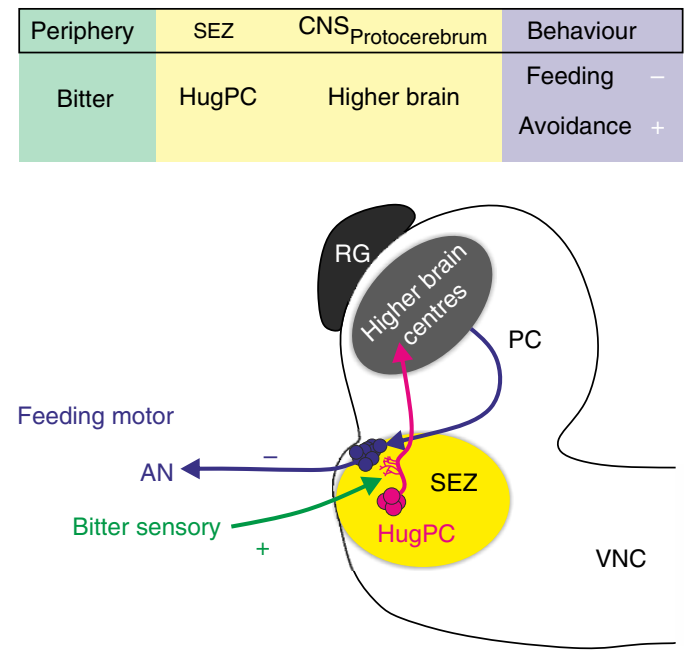

Figure 8 | HuginPC neurons act as gustatory interneurons for bitter taste. Bitter taste, detected in peripheral taste organs, activates huginPC neurons. Activity of huginPC neurons projecting to higher brain centres leads to a decrease in food intake and in the cycle frequency of feeding related motor patterns in the antennal nerve (AN). Activation of huginPC neurons also leads to aversion to different gustatory substrates, including yeast, thereby triggering aversive behaviour. Thus, huginPC neurons act as relay of bitter taste to the protocerebrum in the Drosophila larval brain.

controller (Thorlabs, LEDDB1 driven with $1000 \mathrm{~mA}$ ) and positioned $18 \mathrm{~cm}$ above the solution with the larva. PCR plate of 96 -well was filled with $50 \mu \mathrm{l}$ containing the given taste stimuli in tap water. Larvae were placed into the solution for $2 \mathrm{~min}$ and then ultraviolet light was applied for $30 \mathrm{~s}$.

Afterwards brains were dissected in PBS and mounted onto a Poly-L-lysine (Sigma, Lot \# SLBG4596V) coated cover slide with a drop of PBS. All z-stacks of the HugPC neurons were aquired using a ZEISS LSM 780 Laser scanning microscope with LCI Plan-Neofluar $25 \times / 0.8$ Imm Korr DIC M27. For quantification of green to red photoconversion maximum intensity projections of the acquired $z$-stacks were used and a portion of the cytoplasmatic region of each cell was analysed to obtain data for green and red fluorescence intensity. Red fluorescence intensity was divided by green fluorescence intensity to get $\mathrm{F}_{\text {red }} / \mathrm{F}_{\text {green }}$ ratio. A 'no ultraviolet light' control was included to show that scanning of the CNS without being exposed to ultraviolet light does not convert green to red fluorescence.
GRASP. Genotypes used for GFP reconstitution across synaptic partners (GRASP) method $^{19}$ were: Hug1.2lexA;lexAop-CD4::spGFP11 and GR66a-Gal4;UASCD4::spGFP1-10. Larval CNS was dissected and stained with anti-mouse-GFP (Abcam, 1:500, secondary antibody was anti-mouse-Al488 (Invitrogen, 1:500)). Images were acquired using a ZEISS LSM 780 Laser scanning microscope with LCI Plan-Neofluar $25 \times$ per 0.8 Imm Korr DIC M27.

Calcium imaging with GCaMP. Freshly dissected CNS of feeding third instar larvae of the genotypes UAS-dTrpA1/Hug1.2lexA; GR66a-Gal4/lexAop-GCamP6sp10 (experiment) and Hug1.2lexA/CyO; lexAop-GCamP6s/TM3, Sb (control) were placed with the SEZ region up on a Poly-L-lysine coated coverslide in a drop of saline. Coverslide was attached to a custom built heating device consisting of a $1.5 \mathrm{~cm}^{2}$ Peltier element for shifting the temperature from 20 to $30^{\circ} \mathrm{C}$ by applying specific voltage values. Images were acquired with a Zeiss LSM 780 laser scanning microscope as time series with a speed of $781.96 \mathrm{~ms}(\sim 1.28 \mathrm{~Hz})$ using a Zeiss LCI 'Plan-Neofluar' $25 \times$ per 0.8 Imm Korr DIC M27 objective dipped in the saline solution. Region of interest covered the complete HugPC neuronal 'sprinkler-like' arborization pattern in the protocerebrum.

Statistics. For comparison of two groups in the two choice assays Mann-WhitneyRank-Sum-Test was used. Statistical data were acquired as cumulative PI values of the last $5 \mathrm{~min}$ of the $20 \mathrm{~min}$ experiment and displayed as boxplots. For food intake analysis $32{ }^{\circ} \mathrm{C}$ values (\% of red yeast in gut relative to whole body) were divided by the mean of all $18^{\circ} \mathrm{C}$ values to gather the fold change of food intake. Fold changes were then compared with the Mann-Whitney-Rank-sum-Test. For electrophysiological data cycle frequencies were analysed at $18^{\circ} \mathrm{C}$ for $60 \mathrm{~s}$ and $32^{\circ} \mathrm{C}$ for $60 \mathrm{~s}$. The fold change was calculated between 18 and $32^{\circ} \mathrm{C}$. Per larva a maximum of four temperature steps could be applied to the CNS during recordings. All fold changes of one genotype were then compared with the Mann-Whitney-Rank-SumTest with the other genotypes. The relative fold change is displayed as subtraction of the mean of $\operatorname{OrgR} \times \mathrm{dTrpAl}$ control values from the fold change values of all genotypes $(\operatorname{OrgR} \times \mathrm{dTrpA} 1$ set to 0$)$. CaMPARI data were analysed by calculating the mean $\mathrm{F}_{\text {red }} / \mathrm{F}_{\text {green }}$ value of all eight HuginPC neurons of one larva to determine one mean $\mathrm{F}_{\text {red }} / \mathrm{F}_{\text {green }}$ value per larva. Values were then compared with the Mann-Whitney-Rank-Sum-Test to water control.

Data availability. The authors declare that the data supporting the findings of this study are available within the article (and its Supplementary Information files), or available from the authors upon request.

\section{References}

1. Kwon, J. Y., Dahanukar, A., Weiss, L. A. \& Carlson, J. R. Molecular and cellular organization of the taste system in the Drosophila larva. J. Neurosci. 31, 15300-15309 (2011).

2. Apostolopoulou, A. A., Rist, A. \& Thum, A. S. Taste processing in Drosophila larvae. Front. Integr. Neurosci. 9, 1-9 (2015). 
3. Breslin, P. A. S. \& Spector, A. C. Mammalian taste perception. Curr. Biol. 18, R148-R155 (2008).

4. Yarmolinsky, D. A., Zuker, C. S. \& Ryba, N. J. P. Common sense about taste: from mammals to insects. Cell 139, 234-244 (2009).

5. Joseph, R. M. \& Carlson, J. R. Drosophila chemoreceptors: a molecular interface between the chemical world and the brain. Trends Genet. 31, 683-695 (2015).

6. Clyne, P. J. Candidate taste receptors in Drosophila. Science 287, 1830-1834 (2000).

7. Scott, K. et al. A chemosensory gene family encoding candidate gustatory and olfactory receptors. Cell 104, 661-673 (2001).

8. Dunipace, L., Meister, S., McNealy, C. \& Amrein, H. Spatially restricted expression of candidate taste receptors in the Drosophila gustatory system. Curr. Biol. 11, 822-835 (2001).

9. Weiss, L. A., Dahanukar, A., Kwon, J. Y., Banerjee, D. \& Carlson, J. R. The molecular and cellular basis of bitter taste in Drosophila. Neuron 69, 258-272 (2011).

10. Harris, D. T., Kallman, B. R., Mullaney, B. C. \& Scott, K. Representations of taste modality in the Drosophila brain. Neuron 86, 1449-1460 (2015).

11. Carleton, A., Accolla, R. \& Simon, S. A. Coding in the mammalian gustatory system. Trends Neurosci. 33, 326-334 (2010).

12. Kain, P. \& Dahanukar, A. Secondary taste neurons that convey sweet taste and starvation in the Drosophila brain. Neuron 85, 819-832 (2015).

13. Melcher, C. \& Pankratz, M. J. Candidate gustatory interneurons modulating feeding behavior in the Drosophila brain. PLoS Biol. 3, e305 (2005).

14. Bader, R. et al. Genetic dissection of neural circuit anatomy underlying feeding behavior in Drosophila: distinct classes of hugin-expressing neurons. J. Comp. Neurol. 502, 848-856 (2007).

15. Marella, S. et al. Imaging taste responses in the fly brain reveals a functional map of taste category and behavior. Neuron 49, 285-295 (2006).

16. Moon, S. J., Köttgen, M., Jiao, Y., Xu, H. \& Montell, C. A taste receptor required for the caffeine response in vivo. Curr. Biol. 16, 1812-1817 (2006).

17. Thorne, N., Chromey, C., Bray, S. \& Amrein, H. Taste perception and coding in Drosophila. Curr. Biol. 14, 1065-1079 (2004).

18. Hernandez-Nunez, L. et al. Reverse-correlation analysis of navigation dynamics in Drosophila larva using optogenetics. Elife 4, 1-4 (2015).

19. Gordon, M. D. \& Scott, K. Motor control in a Drosophila taste circuit. Neuron 61, 373-384 (2009).

20. Schoofs, A. et al. Selection of motor programs for suppressing food intake and inducing locomotion in the Drosophila brain. PLoS Biol. 12, e1001893 (2014).

21. Niewalda, T. et al. Salt processing in larval Drosophila: choice, feeding, and learning shift from appetitive to aversive in a concentration-dependent way. Chem. Senses 33, 685-692 (2008).

22. Fosque, B. F. et al. Labeling of active neural circuits in vivo with designed calcium integrators. Science 347, 755-760 (2015).

23. Chu, B., Chui, V., Mann, K. \& Gordon, M. D. Presynaptic gain control drives sweet and bitter taste integration in Drosophila. Curr. Biol. 24, 1978-1984 (2014).

24. König, C., Schleyer, M., Leibiger, J., El-Keredy, A. \& Gerber, B. Bitter-sweet processing in larval Drosophila. Chem. Senses 40, 445-445 (2015).

25. Peng, Y. et al. Sweet and bitter taste in the brain of awake behaving animals. Nature 527, 512-515 (2015).

26. French, A. S. et al. Dual mechanism for bitter avoidance in Drosophila. J. Neurosci. 35, 3990-4004 (2015).

27. Meunier, N., Marion-Poll, F., Rospars, J.-P. \& Tanimura, T. Peripheral coding of bitter taste in Drosophila. J. Neurobiol. 56, 139-152 (2003).
28. Alves, G., Sallé, J., Chaudy, S., Dupas, S. \& Manière, G. High-NaCl perception in Drosophila melanogaster. J. Neurosci. 34, 10884-10891 (2014).

29. Zhang, Y. V., Ni, J. \& Montell, C. The molecular basis for attractive salt-taste coding in Drosophila. Science 340, 1334-1338 (2013).

30. Nakamura, M., Baldwin, D., Hannaford, S., Palka, J. \& Montell, C. Defective proboscis extension response (DPR), a member of the Ig superfamily required for the gustatory response to salt. J. Neurosci. 22, 3463-3472 (2002).

31. Wang, Z., Singhvi, A., Kong, P. \& Scott, K. Taste representations in the Drosophila brain. Cell 117, 981-991 (2004).

32. Bader, R. et al. The IGFBP7 homolog Imp-L2 promotes insulin signaling in distinct neurons of the Drosophila brain. J. Cell Sci. 126, 2571-2576 (2013).

33. Zhou, L. et al. Cooperative functions of the reaper and head involution defective genes in the programmed cell death of Drosophila central nervous system midline cells. Proc. Natl. Acad. Sci. 94, 5131-5136 (1997).

34. Kitamoto, T. Conditional modification of behavior in Drosophila by targeted expression of a temperature-sensitive Shibire allele in defined neurons. J. Neurobiol. 47, 81-92 (2001).

\section{Acknowledgements}

We thank Ingo Zinke and Andreas Schoofs for comments and discussions on the manuscript, Andreas Schoofs for help with electrophysiology, Philipp Schlegel and Anton Miroschnikow for sharing analysis scripts, Marius Wegner for reagents and Daniela Müller for help with behavioural experiments. We thank Kristin Scott, Andreas Thum and the Bloomington stock centre for sharing fly lines. This work was supported by grants from DFG (PA 787/7-1) to MJP and Bonn Cluster of Excellence ImmunoSensation.

\section{Author contributions}

S.H. and M.J.P. designed experiments and wrote the manuscript. S.H. carried out the experiments and analysed the data. M.P. performed the GRASP experiment.

\section{Additional information}

Supplementary Information accompanies this paper at http://www.nature.com/ naturecommunications

Competing financial interests: The authors declare no competing financial interests.

Reprints and permission information is available online at http://npg.nature.com/ reprintsandpermissions/

How to cite this article: Hückesfeld, S. et al. Central relay of bitter taste to the protocerebrum by peptidergic interneurons in the Drosophila brain. Nat. Commun. 7:12796 doi: 10.1038/ncomms12796 (2016).

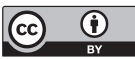

This work is licensed under a Creative Commons Attribution 4.0 International License. The images or other third party material in this article are included in the article's Creative Commons license, unless indicated otherwise in the credit line; if the material is not included under the Creative Commons license, users will need to obtain permission from the license holder to reproduce the material. To view a copy of this license, visit http://creativecommons.org/licenses/by/4.0/

(C) The Author(s) 2016 